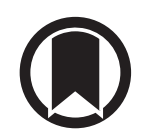

CrossMark

\title{
The pulmonary haemodynamics during exercise - research network (PEX-NET) ERS Clinical Research Collaboration: investigating the prognostic relevance of exercise haemodynamics
}

\author{
Gabor Kovacs ${ }^{1,2}$, Philippe Herve ${ }^{3}$ and Horst Olschewski $i^{1,2}$ on behalf of the \\ PEX-NET Clinical Research Collaboration
}

Affiliations: ${ }^{1}$ Medical University of Graz, Graz, Austria. ${ }^{2}$ Ludwig Boltzmann Institute for Lung Vascular Research, Graz, Austria. ${ }^{3}$ Centre Chirugical Marie Lannelongue, Thoracic and Vascular Surgery, Le Plessis-Robinson, France.

Correspondence: Gabor Kovacs, Medical University of Graz, Auenbruggerplatz 20, 8036 Graz, Austria. E-mail: gabor.kovacsaklinikum-graz.at

@ERSpublications

PEX-NET is likely to provide answers regarding the prognostic relevance of pulmonary haemodynamics during exercise and will stimulate further research on the underlying pathophysiology, diagnosis and treatment of exercise pulmonary hypertension http://ow.ly/VThP30o2Anl

Cite this article as: Kovacs G, Herve P, Olschewski H. The pulmonary haemodynamics during exercise research network (PEX-NET) ERS Clinical Research Collaboration: investigating the prognostic relevance of exercise haemodynamics. Eur Respir J 2019; 53: 1900458 [https://doi.org/10.1183/13993003.00458-2019].

\section{Background and rationale}

An elevation of mean pulmonary arterial pressure (PAP) at rest represents a severe medical condition that is independently associated with increased mortality [1-3]. In subjects with normal PAP at rest, an abnormal increase during exercise in relation to the increase in pulmonary arterial blood flow has been proposed as a condition termed "exercise pulmonary hypertension" (exercise PH) [4]. Currently available data support a definition for exercise $\mathrm{PH}$ as an increase in the mean PAP to $>30 \mathrm{mmHg}$ combined with an increase in total pulmonary resistance to $>3$ Wood Units during maximal exercise [5]. Exercise PH may represent an early stage of pulmonary vascular disease and other pathological mechanisms in the heart and/or in the lungs. All these mechanisms may lead to dyspnoea on exertion, which is one of the most worrying symptoms in respiratory medicine.

Pulmonary haemodynamics during exercise became one of the major issues during the fourth $\mathrm{PH}$ world conference in Dana Point (CA, USA) in 2008. Until this conference, PH had been defined as a resting mean PAP $>25 \mathrm{mmHg}$ or an exercise mean PAP $>30 \mathrm{mmHg}$ [6]. Based on a large meta-analysis of almost 1200 healthy subjects it became evident, however, that the normal response of pulmonary haemodynamics to exercise is dependent on both age and the level of exercise and, therefore, no single pressure threshold can be defined to characterise a pathological condition [7]. Accordingly, the exercise part of the $\mathrm{PH}$ definition was abandoned and additional studies were suggested. The conference stated that an updated exercise part of the definition may be re-implemented into the definition of $\mathrm{PH}$ if there is convincing evidence for an association to relevant end-points. At the fifth PH world conference in Nice (France) in 
2013, the possible definition of exercise PH was discussed again. Although single-centre investigations highlighted the clinical relevance of pulmonary haemodynamics during exercise [8], a re-introduction of an exercise definition was not considered.

Subsequently, a European Respiratory Society (ERS) Task Force was implemented, including experts from Europe, Australia and the USA, discussing the current knowledge on pulmonary haemodynamics during exercise. The Task Force results have recently been published [4], summarising the available evidence in the field and highlighting the need for solid data on the prognostic relevance of exercise $\mathrm{PH}$. At the sixth PH world conference in Nice in 2018, a change in the definition of resting PH was decided. However, a definition for "exercise $\mathrm{PH}$ " was not re-introduced [1], due to the lack of large-scale, multicentre, long-term data regarding prognostic implications, as pointed out by the ERS Task Force.

Recently, the ERS funded such a large-scale multicentre approach by using retrospective and prospective data of haemodynamics at rest and exercise to analyse the prognostic implications of exercise PH. This Clinical Research Collaboration (CRC) [9] is called the "pulmonary haemodynamics during exercise research network" (PEX-NET) and is mainly supported by the ERS assemblies 13 (pulmonary vascular diseases) and 4 (group 04.01 clinical respiratory physiology, exercise and functional imaging).

\section{PEX-NET aims}

The main goal of PEX-NET is to investigate the prognostic relevance of pulmonary haemodynamics during exercise, as assessed by right heart catheterisation in patients undergoing this procedure due to clinical reasons, and to identify independent predictors of relevant clinical end-points. The primary end-point is mortality/lung transplantation, and the secondary end-points include hospitalisation, the development of $\mathrm{PH}$ at rest or the initiation of targeted pulmonary arterial hypertension (PAH) medication. Importantly, we aim to investigate the prognostic information of pulmonary haemodynamics during exercise on top of all the clinical information (assessed at rest) that is already available for the patients. This registry study does not intend to influence individual diagnostic or treatment decisions in any way. Relevant clinical events will be captured and documented.

\section{What PEX-NET has achieved so far}

The web-based PEX-NET database has been developed in close collaboration with the Institute for Medical Informatics, Statistics and Documentation at the Medical University of Graz (Graz, Austria). The database has been developed in Clincase, a web-based, 21 CFR (Code of Federal Regulations) 11-compliant electronic data capture system for multicentre clinical trials. The database has the following main parts: 1) centre-specific data regarding the performance of right heart catheterisation and the assessment of haemodynamics during exercise; 2) patient data, including patient characteristics, comorbidities and additional investigations; 3 ) haemodynamic data assessed by right heart catheterisation at rest and during exercise; and 4) patient outcome data on mortality/lung transplantation, hospitalisation, the development of $\mathrm{PH}$, the initiation of targeted $\mathrm{PAH}$ therapy and the last date of observation.

For the retrospective part of the registry, data entry began at the end of 2018 and we expect that over 1000 patients will be included (all available patients with clinically indicated right heart catheterisation with exercise haemodynamics and sufficient follow-up data from the participating centres). Similarly in the prospective part, the first patients were included at the end of 2018. In these individuals, study end-points will be prospectively documented for a planned duration of 5 years. The planned number of patients in the prospective part is 498 . The main elements of a centre-specific haemodynamic protocol are shown in table 1. Thus, the PEX-NET CRC will create a highly flexible database for capturing data from different laboratories with diverse devices and protocols.

\section{The potential of PEX-NET and future steps}

There are several potential major long-term benefits of the PEX-NET CRC. First, we hope that PEX-NET will provide the evidence that is needed to re-implement a definition for exercise $\mathrm{PH}$ in one of the next $\mathrm{PH}$ world conferences and ignite further diagnostic and therapeutic studies in the field. Secondly, after describing current evidence on pulmonary haemodynamics and identifying knowledge gaps [4], and addressing the most important arising clinical question (prognostic relevance) in a prospective, multicentre manner, we also aim for a better understanding of the pathophysiology of exercise $\mathrm{PH}$. This may start with the description of the scientific evidence in the field of pulmonary capillary recruitment, focusing on the main physiological concepts and the methods for investigating pulmonary capillary recruitment, as well as findings on pulmonary capillary recruitment from experimental and clinical research studies. Addressing these issues is highly relevant as it is essential for an appropriate diagnostic interpretation of pulmonary haemodynamic responses to exercise. Thirdly, discussion of methodological details of the assessment of pulmonary haemodynamics during exercise within the CRC may help to 
TABLE 1 The main elements of a centre-specific haemodynamic protocol

\section{Patient position \\ Achieved maximal exercise \\ Exercise method \\ Zero reference level}

\author{
Determination of cardiac \\ output \\ Determination of pressure \\ values \\ Transition from rest to \\ exercise
}

Supine, semi-supine or upright

Maximal effort, or other

Cycle ergometry, or other

Mid-thoracic level in the supine patient, the intersection of the frontal plane at the mid-thoracic level, the transverse plane at the level of the fourth anterior intercostal space, and the mid-sagittal plane in the semi-supine patient, or other

Direct Fick method or thermodilution

Averaged over 3-5 respiratory cycles, or other

Changes in any relevant condition (position, zero level, determination of pressures, breathing manoeuvres, cardiac output assessment)

provide a standardised approach that can promote reproducibility of results among different centres. Finally, PEX-NET provides a scientific and clinical platform for investigators and clinical researchers assessing pulmonary haemodynamics during exercise and may stimulate participants of the CRC to perform further studies, initiate new collaborations and submit proposals for additional funding.

Expert centres with extensive experience in the treatment of patients with $\mathrm{PH}$ and performance of right heart catheterisations during exercise may join this CRC. Decisions will be made on an individual basis by the Chairs and the Steering Committee of the CRC.

In conclusion, PEX-NET is likely to provide answers regarding the prognostic relevance of pulmonary haemodynamics during exercise and will stimulate further research on the underlying pathophysiology of exercise $\mathrm{PH}$ as well as on the diagnostic and therapeutic options for patients.

Conflict of interest: G. Kovacs reports personal fees and non-financial support from Actelion, Bayer, GSK, MSD, AOP, Boehringer Ingelheim, Novartis and Chiesi, outside the submitted work. P. Herve has nothing to disclose. H. Olschewski reports grants from Bayer, Unither Pharmaceuticals, Actelion Pharmaceuticals Ltd, Roche, Boehringer Ingelheim and Pfizer Inc., personal fees from Gilead Sciences Inc., Encysive Pharmaceuticals Ltd and Nebu-Tec, and personal fees and non-financial support from Bayer, Unither Pharmaceuticals, Actelion Pharmaceuticals Ltd, Pfizer Inc., Eli Lilly, Novartis, AstraZeneca, Boehringer Ingelheim, Chiesi, Menarini, MSD and GSK, outside the submitted work.

Support statement: Funding was received from the European Respiratory Society. Funding information for this article has been deposited with the Crossref Funder Registry.

\section{References}

1 Simonneau G, Montani D, Celermajer DS, et al. Haemodynamic definitions and updated clinical classification of pulmonary hypertension. Eur Respir J 2019; 53: 1801913.

2 Douschan P, Kovacs G, Avian A, et al. Mild elevation of pulmonary arterial pressure as a predictor of mortality. Am J Respir Crit Care Med 2018; 197: 509-516.

3 Maron BA, Hess E, Maddox TM, et al. Association of borderline pulmonary hypertension with mortality and hospitalization in a large patient cohort: insights from the Veterans Affairs Clinical Assessment, Reporting, and Tracking program. Circulation 2016; 133: 1240-1248.

4 Kovacs G, Herve P, Barbera JA, et al. An official European Respiratory Society statement: pulmonary haemodynamics during exercise. Eur Respir J 2017; 50: 1700578.

5 Herve P, Lau EM, Sitbon O, et al. Criteria for diagnosis of exercise pulmonary hypertension. Eur Respir J 2015; 46: $728-737$.

6 Galiè N, Torbicki A, Barst R, et al. Guidelines on diagnosis and treatment of pulmonary arterial hypertension. The Task Force on Diagnosis and Treatment of Pulmonary Arterial Hypertension of the European Society of Cardiology. Eur Heart J 2004; 25: 2243-2278.

7 Kovacs G, Berghold A, Scheidl S, et al. Pulmonary arterial pressure during rest and exercise in healthy subjects: a systematic review. Eur Respir J 2009; 34: 888-894.

8 Naeije R, Vanderpool R, Dhakal BP, et al. Exercise-induced pulmonary hypertension: physiological basis and methodological concerns. Am J Respir Crit Care Med 2013; 187: 576-583.

9 Brightling C, Genton C, Bill W, et al. ERS Clinical Research Collaborations: underpinning research excellence. Eur Respir J 2018; 52: 1801534. 